\title{
Application of a Modified Arrhenius Equation to Describe the Time-Temperature Equivalence in Relaxation Analysis of Metal Seals
}

\author{
Linan Qiao, Sven Nagelschmidt and Uwe Herbrich \\ Bundesanstalt für Materialforschung und -prüfung (BAM), 12200 Berlin, Germany
}

\begin{abstract}
For the application of the time-temperature superposition principle a suitable relation is needed to describe the time-temperature shift factor $\alpha$. Therefore, the Arrhenius equation is widely used due to its simple form and often leads to suitable results. Where, the Arrhenius equation presents a linear relation for the temperature-dependent shift factor in logarithmic scale $\ln (\alpha)$ with the absolute inverse temperature $(1 / \vartheta)$. However, in cases with a large temperature range which eventually include more complex reaction processes, the functional relation between $\ln (\alpha)$ and $(1 / \vartheta)$ is nonlinear in the 'Arrhenius plot'. In those cases, the monotone change of the nonlinear range in the 'Arrhenius plot' can be interpreted as a transient range between two approximately linear or constant regions. An extended application of the modified Arrhenius equation from Nakamura (1989) is presented in this study for this transient range. The introduced method was applied to describe the time-temperature equivalence in the relaxation analysis of restoring seal force of metal seals, which are used in lid-systems of transport and interim storage casks for radioactive materials. But, the method is widely valid and can be used for different objectives which are characterized by thermorheologically simple behavior with nonlinear sensitivity to inverse temperature.
\end{abstract}

Key words: TTS principle, Arrhenius equation, non-linear Arrhenius behavior, relaxation analysis, seal force, metal seals.

\section{Introduction}

Metal seals are widely used in bolted lid-systems to ensure appropriate leak-tightness for several decades, e.g., for the confinement of casks for transport and interim storage of radioactive materials. Mechanical properties of metal seals change with time and those changes are depending on temperature during the long-term service, especially due to the thermo-viscoplastic deformation of the outer jacket made of aluminum or silver. Hence, the restoring seal force decreases with time and its rate is very sensitive to temperature.

A phenomenological-analytical model for metal seals of type HELICOFLEX ${ }^{\circledR}$ HN200, see Fig. 1, has been developed recently [1], to describe the time and

Corresponding author: Linan Qiao, Ph.D., engineer, research fields: material science, safety analyse of transport and storage containers of radioactive materials. E-mail: linan.qiao@bam.de. temperature dependent decrease of the seal force. This model focuses on the global mechanical behavior of metal seals and considers all seal components together as a 'homogeneous material block' in contrast to a detailed two- or three-dimensional finite element model of metal seals considering each seal component [2]. The development of the model is based on experimental results derived from investigations which have been carried out at the Bundesanstalt für Materialforschung und -prüfung (BAM), a federal institute for materials research and testing in Germany, since 2009, see past works in Refs. [3-5].

Experimental findings are shown in Fig. 2 with different point styles for seals with aluminum outer jacket (Al-seals) and seals with silver outer jacket (Ag-seals). The seals are tested in the absolute temperature range between $293 \mathrm{~K}$ and $423 \mathrm{~K}$.

The restoring seal force $Y_{2 R}$ is specified as the remaining length-related force $Y=F /(\pi D)$, with seal 


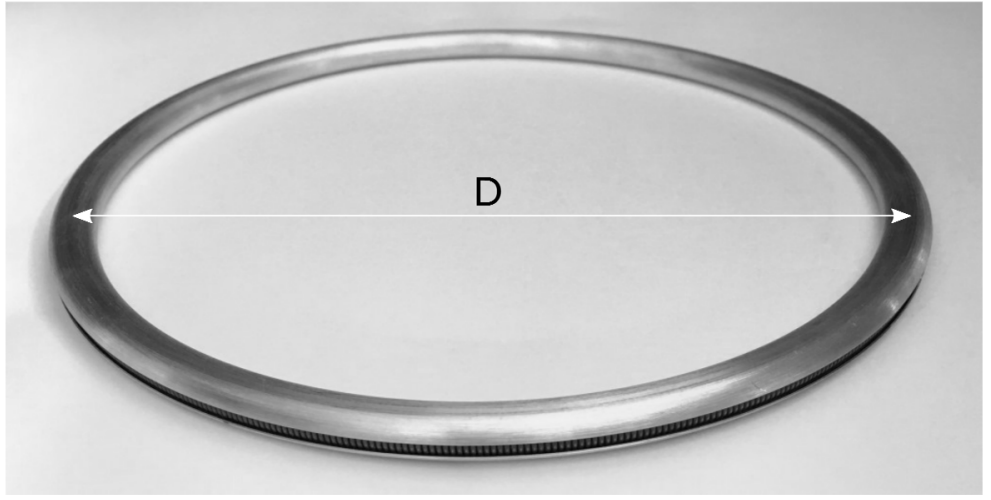

(a)

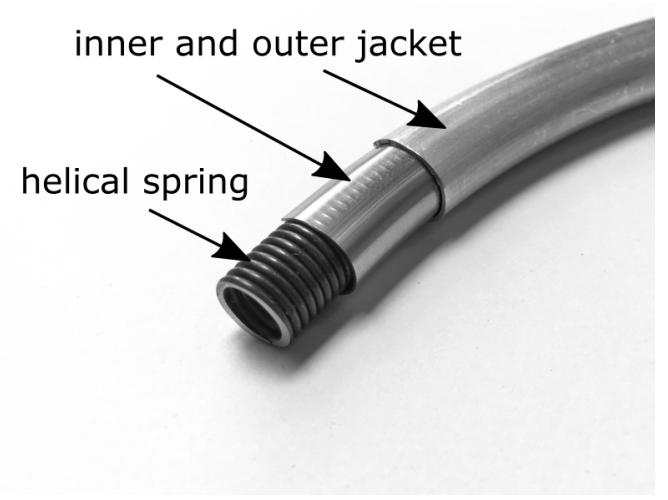

(b)

Fig. 1 Investigated metal seal: (a) test specimen; and (b) labelled cross section view.

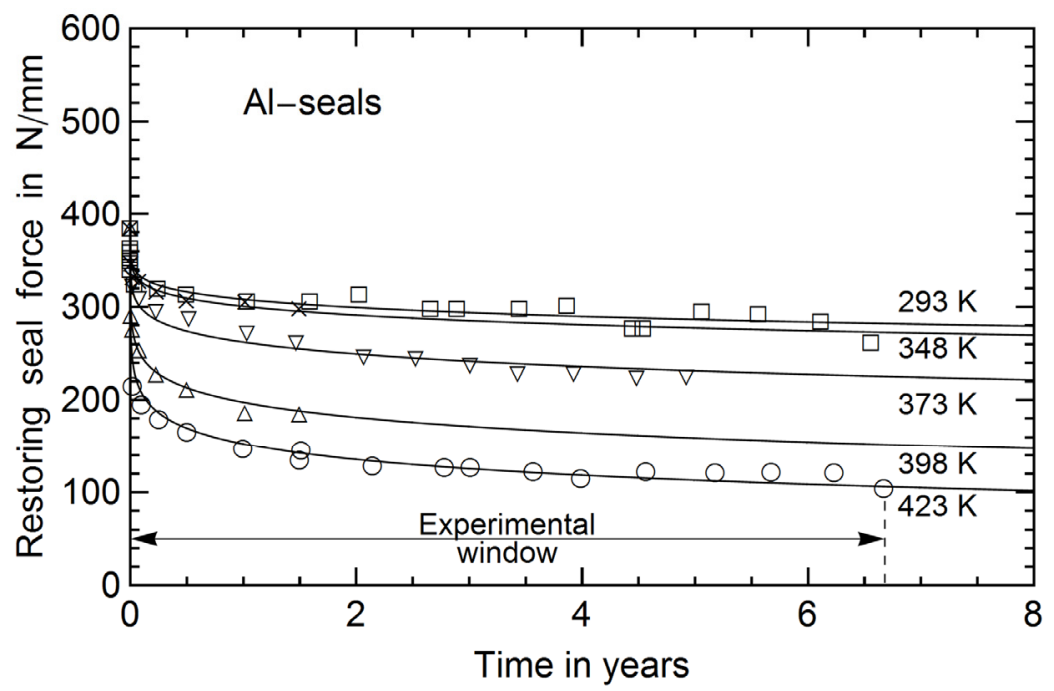

(a)

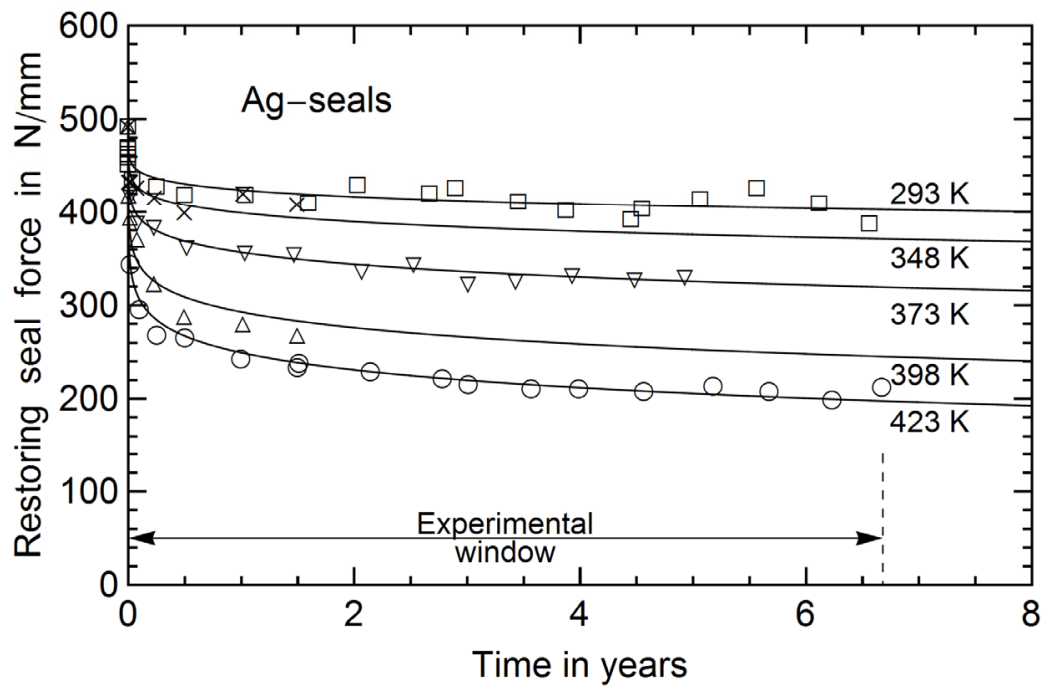

(b)

Fig. 2 Restoring seal forces of investigated metal seals with: (a) Al-seals; and (b) Ag-seals for five different constant test temperatures. 
force $Y$ in $\mathrm{N} / \mathrm{mm}$, overall force $F$ in $\mathrm{N}$ and seal diameter $D$ in $\mathrm{mm}$.

The restoring seal force $Y_{2 R}$ decreases with time and the decrease is accelerated with increasing temperature, especially in the first year of tests.

In Fig. 2, the continuous lines present the calculation results of a prior developed 'enhanced power-law model' with different test temperatures, see Ref. [1]. Focus of this study is to develop an analytic function which can be used to calculate the restoring seal force with time under arbitrary constant temperatures in the studied temperature range between $293 \mathrm{~K}$ and $423 \mathrm{~K}$. For this purpose, a modified Arrhenius equation from Nakamura et al. [6] will be extended and applied for studied metal seals under consideration of the time-temperature superposition principle (TTS) .

\section{Describing Seal Relaxation Behavior with TTS Principle}

With the assumption that the rate of force relaxation of metal seals is proportional to seal force itself and inverse in time with a constant temperature-dependent exponent as $\dot{Y}_{2 R, E}=c \cdot Y_{2 R, E} \cdot t^{-m}$, the relaxation behavior of metal seals can be suitably described by using an 'enhanced power-law model' for each test temperature in the form:

$$
Y_{2 R, E}=Y_{\text {init }} \cdot \exp \left[\ln \left(\frac{Y_{r e f, E}}{Y_{\text {init }}}\right) \frac{\left(\tau^{1-m}-\tau_{\text {init }}^{1-m}\right)}{\left(1-\tau_{\text {init }}^{1-m}\right)}\right]
$$

as introduced in the previous work [1]. In Eq. (1), $\tau=t / t_{\text {ref }}$ is the normalized relaxation time with $t_{\text {ref }}=1$ year, $\tau_{\text {init }}=t_{\text {inil }} / t_{\text {ref }}$ is the normalized start time of the relaxation calculation. Eq. (1) satisfies the conditions $\left.\quad Y_{2 R}\right|_{\tau=\tau_{\text {init }}}=Y_{\text {init }} \quad,\left.\quad Y_{2 R}\right|_{\tau=1}=Y_{\text {ref }, E} \quad$ and $\left.Y_{2 R}\right|_{\tau \rightarrow \infty}=0$ as a convergent function. The authors use $\tau_{\text {init }}=0$ with $Y_{\text {init }}=Y_{2, A l}$ for Al-seals and $Y_{\text {init }}=Y_{2, A g}$ for Ag-seals by the fact that all seals were compressed initially at room temperature and the decreasing of the seal force starts just after the initial compression. Parameters $Y_{\text {ref,E }}$ and $m$ are temperature-dependent. If $\tau_{\text {init }}$ is zero, Eq. (1) has a similar form of a stretched exponential function. Models with those 'enhanced power-law relations' are often used for a phenomenological description of relaxation behavior, which was introduced by Kohlrausch [7] and Williams and Watts [8] and is applied for metal seals, see Ref. [1].

For the application of TTS, Eq. (1) must be modified to safe the necessary condition of this principle. For this purpose, a 'mean enhanced power-law model' was developed for the determination of a master function under consideration of TTS, where a temperatureindependent constant $\bar{m}$ as a mean value of the temperature dependent parameters of $m\left(\vartheta_{i}\right)$ for each test temperature in the form

$$
\bar{m}=\sum_{i=1}^{5} m\left(\vartheta_{i}\right) / 5
$$

was used for the mean enhanced power-law model:

$$
\bar{Y}_{2 R, E}=Y_{2} \cdot \exp \left[\ln \left(\frac{\bar{Y}_{r e f, E}\left(\vartheta_{r e f}\right)}{Y_{2}}\right)\left(\alpha_{E}\left(\vartheta_{i}\right) \tau\right)^{1-\bar{m}}\right]
$$

In Eq. (2), $\bar{Y}_{2 R, E}$ is the restoring seal force calculated from the 'mean enhanced power-law model', $Y_{2}$ is the initial seal force at $\tau=0$ and $\bar{Y}_{r e f, E}\left(\vartheta_{\text {ref }}\right)$ is the corresponding temperature dependent parameter at $\tau=1$ with constant $\bar{m}$ for the reference temperature $\vartheta_{\text {ref }}=\vartheta_{1}=293 \mathrm{~K}$. Thus, Eq. (2) has only one temperature-dependent multiplication factor $\alpha_{E}\left(\vartheta_{i}\right)$ with time, which satisfies the implicit premise of TTS principle. The time-temperature shift factors $\alpha_{E}\left(\vartheta_{i}\right)$ were calculated from

$$
\alpha_{E}\left(\vartheta_{i}\right)=\left[\frac{\ln \left(\frac{\bar{Y}_{r e f, E}\left(\vartheta_{i}\right)}{Y_{2}}\right)}{\ln \left(\frac{\bar{Y}_{r e f, E}\left(\vartheta_{r e f}\right)}{Y_{2}}\right)}\right]^{\frac{1}{1-\bar{m}}} \quad i=\{1,2 \ldots 5\}
$$

Eq. (3) satisfies the condition of $\alpha_{E}\left(\vartheta_{r e f}\right)=\alpha_{E}\left(\vartheta_{1}\right)=1 \quad$ because the minimal 
temperature of the test range with $\vartheta_{r e f}=\vartheta_{1}$ is chosen in this work. All parameters used for the studied metal seals are given in Table 1.

Eq. (2) with $\vartheta_{i}=\vartheta_{\text {ref }}$ in logarithmic time scale for both seal types is shown in Fig. 3 with continuous lines as a master curve for the relaxation behavior. Measured values from constant temperature test conditions above $293 \mathrm{~K}$ were shifted using Eq. (3).

Table 1 Determined parameters of the mean enhanced power-law model.

\begin{tabular}{lll}
\hline Seal type & Al-seals & Ag-seals \\
\hline $\bar{m}$ & 0.826 & 0.846 \\
$Y_{2}$ in $\mathrm{N} / \mathrm{mm}$ & 386.0 & 494.0 \\
$\bar{Y}_{\text {ref,E}}\left(\vartheta_{1}\right)$ in N/mm & 308.2 & 424.1 \\
$\bar{Y}_{r e f, E}\left(\vartheta_{2}\right)$ in N/mm & 300.7 & 399.1 \\
$\bar{Y}_{r e f, E}\left(\vartheta_{3}\right)$ in N/mm & 262.2 & 356.8 \\
$\bar{Y}_{r e f, E}\left(\vartheta_{4}\right)$ in N/mm & 197.5 & 292.9 \\
$\bar{Y}_{r e f, E}\left(\vartheta_{5}\right)$ in N/mm & 152.6 & 249.4 \\
\hline
\end{tabular}

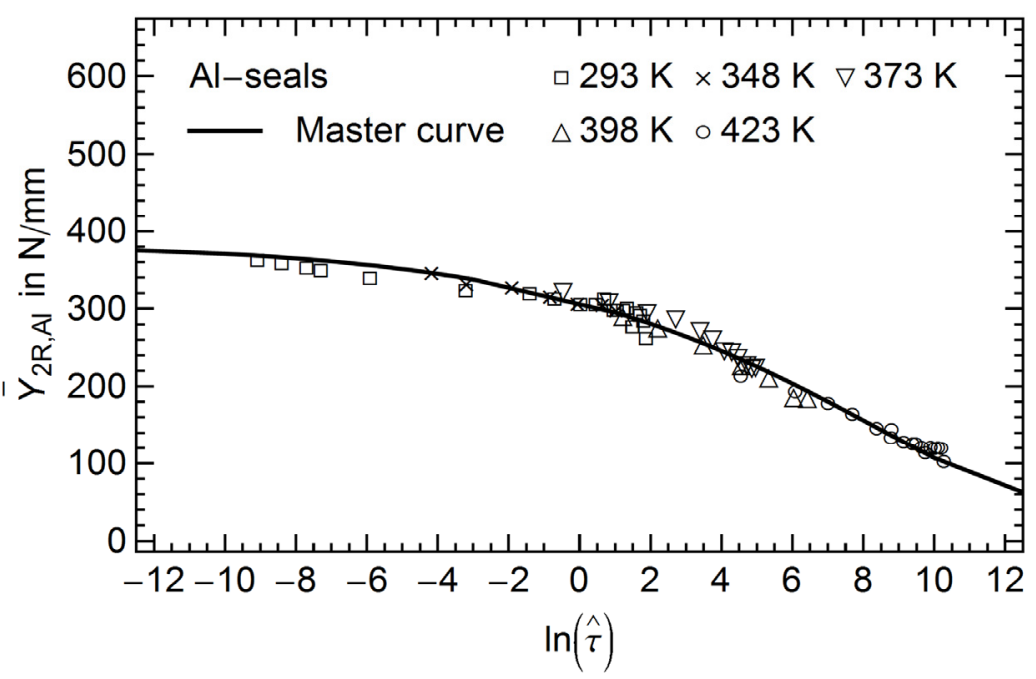

(a)

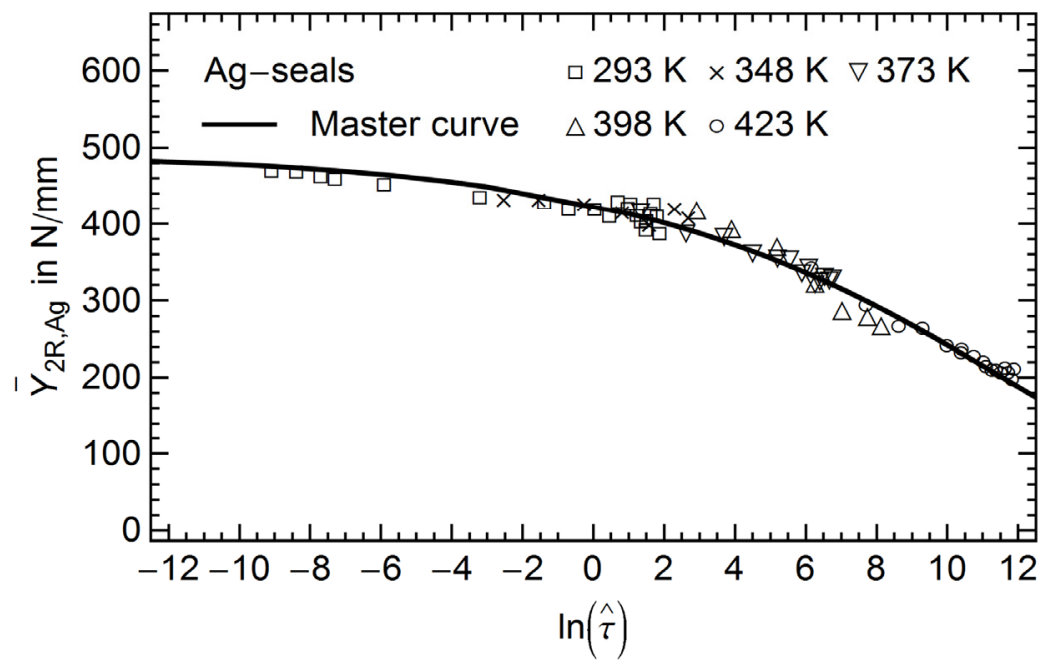

(b)

Fig. 3 Master curve of Eq. (2) and shifted experimental findings with $\alpha_{E}\left(\vartheta_{i}\right)$ from Eq. (3) for different test temperatures for: (a) Al-seals; and (b) Ag-seals, where $\hat{\tau}=\alpha_{E}\left(\vartheta_{i}\right) \tau$ is the master time. 
3. Application of a Modified Arrhenius Equation for the Time-Temperature Shift Factor

The Arrhenius equation [9] is widely used and often leads to suitable results for the continuous description of time-temperature shift factors. But, as shown in Fig. 4, there is no linear relation of Eq. (3) in the plot $\ln (\alpha)$ over normalized inverse temperature $\zeta$ for the entire test temperature range between $293 \mathrm{~K}$ and
$423 \mathrm{~K}$ in case of the 'mean enhanced power-law model' from Eq. (2) is used as a master function. However, two different ranges can be clearly identified: from $293 \mathrm{~K}$ to $348 \mathrm{~K}$ and from $348 \mathrm{~K}$ to $423 \mathrm{~K}$. A linear relation can be approximated for the higher temperature range from $348 \mathrm{~K}$ to $423 \mathrm{~K}$. Furthermore, the non-linear relation of studied metal seals in the 'Arrhenius plot' can be defined as a transient range between two linear or constant ranges.

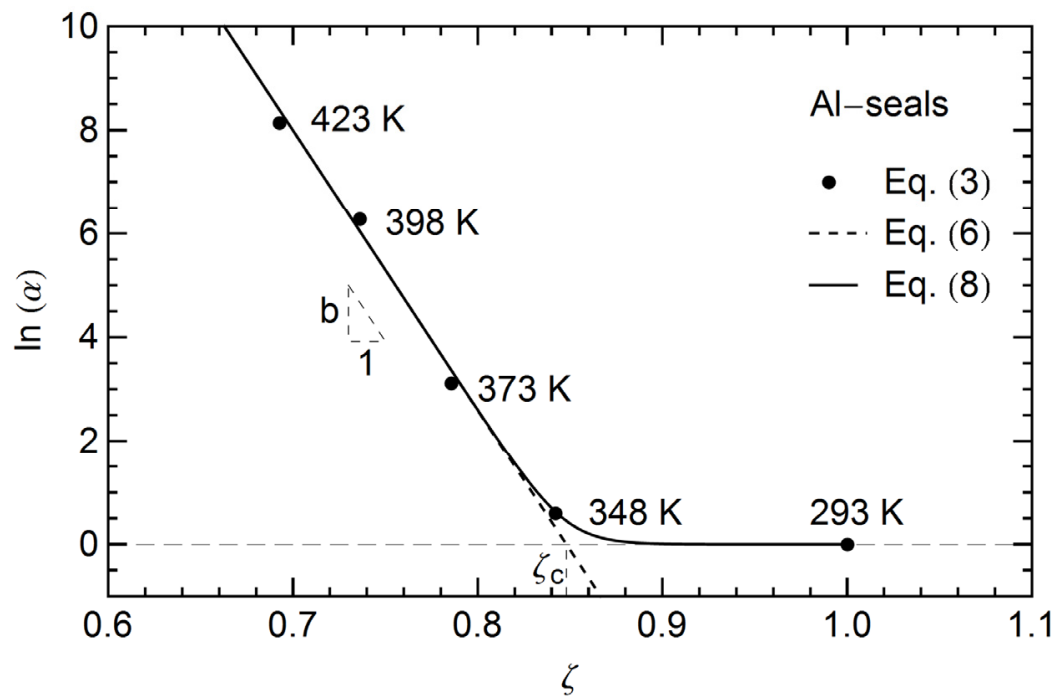

(a)

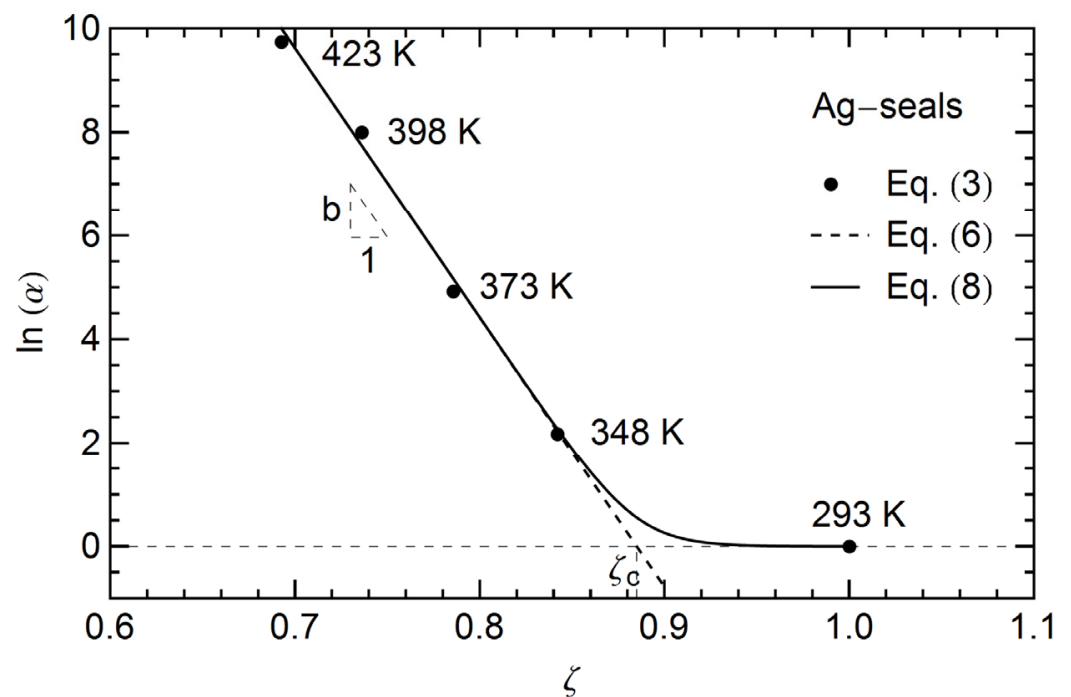

(b)

Fig. 4 Representation of $\alpha_{E}\left(\vartheta_{i}\right)$ from Eq. (3) with dots, $\alpha_{A}(\zeta)$ from Eq. (6) with dashed lines and $\alpha_{N}(\zeta)$ from Eq. (7) with continuous lines for: (a) Al-seals; and (b) Ag-seals. 
Nakamura et al. [6] proposed a modified Arrhenius equation for this transient range. This approach was extended and applied for metal seals in this work At first, the Arrhenius equation was considered, whereat the Arrhenius equation is a simple but general accepted empirical formulation for the description of temperature dependence of reaction rates in the form:

$$
\kappa=A \cdot \exp \left[-\frac{E_{a}}{k_{B} \cdot \vartheta}\right]
$$

with $\kappa$ considered here as the rate of seal force relaxation of metal seals. Additionally, $A$ is the temperature-independent pre-exponential factor and $\vartheta$ is the absolute temperature, $E_{a}$ is the activation energy for the process and was assumed as the equivalent activation energy for the relaxation process of compressed metal seals and $k_{B}$ is the Boltzmann constant. Any temperature rising will result in an increasing reaction rate.

For a temperature $\vartheta_{c}$, as introduced later, the corresponding reaction rate from Eq. (4) is:

$$
\kappa_{c}=A \cdot \exp \left[-\frac{E_{a}}{k_{B} \cdot \vartheta_{c}}\right]
$$

Combining Eqs. (4) and (5), the time-temperature shift factor with the application of the Arrhenius equation is obtained in the form:

$$
\alpha_{A}(\zeta)=\kappa / \kappa_{c}=\exp \left[b\left(\zeta_{c}-\zeta\right)\right]
$$

with $b=\left(E_{a} / k_{B}\right) / \vartheta_{\text {ref }}$.

The quotient $\zeta=\vartheta_{\text {ref }} / \vartheta$ is the absolute inverse temperature normalized with $\vartheta_{\text {ref }}$. The normalized inverse temperature $\zeta_{c}=\vartheta_{\text {ref }} / \vartheta_{c}$ is a parameter and $\zeta=\zeta_{c}$ corresponds to $\alpha_{A}=1$. The meaning of $\zeta_{c}$ will be given later. In Figs. $4 \mathrm{a}$ and $4 \mathrm{~b}$, 'Arrhenius plots' of $\ln (\alpha)$ over normalized inverse temperature $\zeta$ are presented, whereat the dots represent the five different logarithmic shift factors $\ln \left(\alpha_{E}\right)$ from Eq. (3). The doted lines describe the application of Eq. (6) in the range of $348 \mathrm{~K}$ to $423 \mathrm{~K}$.

The results confirm that the Arrhenius equation in its original form can only be used in a limited temperature range, due to the nonlinear relation between $\ln (\alpha)$ and $\zeta$ for the investigated metal seals, for example, for both seal types from approximately $348 \mathrm{~K}$ to $423 \mathrm{~K}$, but not for the complete temperature range of interest from $293 \mathrm{~K}$ to $423 \mathrm{~K}$. Generally, metal seals used in casks are thermally stressed at circa $348 \mathrm{~K}$. Hence, on the one hand, a suitable description of the transient range is necessary and on the other hand the intention of tests at higher temperatures is to accelerate the aging process.

Different modifications of the Arrhenius equation are suggested in the literature, see the work of Laidler [10]. For the description of a nonlinear relation between $\ln (\alpha)$ and $\zeta$, the adjusted modification of the Arrhenius equation proposed by Nakamura et al. [6] in the form:

$$
\kappa_{N}=A \cdot \exp \left[-\frac{E_{a}}{k_{B} \cdot\left(\vartheta^{n}+\vartheta_{c}^{n}\right)^{1 / n}}\right]
$$

was examined as the most suitable form for studied metal seals if 'mean enhanced power-law model' is used as master function. Analog to Eq. (6), the non-linear relation between $\ln (\alpha)$ and $\zeta$ can be get from Eq. (7) in the form

$$
\alpha_{N}(\zeta)=\exp \left\{b \cdot\left[\left(1+\zeta_{c}^{-n}\right)^{-1 / n}-\left(\zeta^{-n}+\zeta_{c}^{-n}\right)^{-1 / n}\right]\right\}
$$

with three parameters $b, \zeta_{c}$ and $n$ that can be easily determined from experimental results.

In Eq. (8), $\zeta_{c}$ is a parameter corresponding to the normalized inverse of an absolute temperature of $\vartheta_{c}$. The parameter $b$ corresponds with the linear gradient of the Arrhenius plot between $\ln (\alpha)$ and $\zeta$ with higher temperatures $(\vartheta>348 \mathrm{~K}$ in this work). Nakamura suggested for the parameter $n$ an amount of 2, but we expanded $n$ to be an arbitrary positive integer.

Eq. (8) describes all test results of metal seals very suitable with relatively large values of $n$, without a 


\section{Application of a Modified Arrhenius Equation to Describe the Time-Temperature Equivalence in Relaxation Analysis of Metal Seals}

detailed explanation of its physical meaning so far. But, from a mathematical point of view, the interaction of $\zeta_{c}$ and the exponent $n$ in Eq. (8) can be explained as follows: $\zeta_{c}$ defines the position of the transient range and $n$ defines the quantity of this range, whereat, the transient range is narrower for a larger $n$. One can obtain exactly two linear ranges for $n \rightarrow \infty: \alpha_{N}=1$ if $\zeta>\zeta_{c}$ and $\alpha_{N}=\alpha_{A}$ if $\zeta \leq \zeta_{c}$, see doted lines in Fig. 4.

Eq. (8) is shown in Fig. 4 with continuous lines and the three determined parameters $b, \zeta_{c}$ and $n$ for the studied metal seals are given in Table 2.

Table 2 Used parameters for the calculation of $\alpha_{N}(\zeta)$ with $\vartheta_{r e f}=293 \mathrm{~K}$.

\begin{tabular}{lll}
\hline Seal type & Al-seal & Ag-seal \\
\hline$b$ & 54 & 52 \\
$\zeta_{c}$ & 0.848 & 0.885 \\
$n$ & 70 & 57 \\
\hline
\end{tabular}

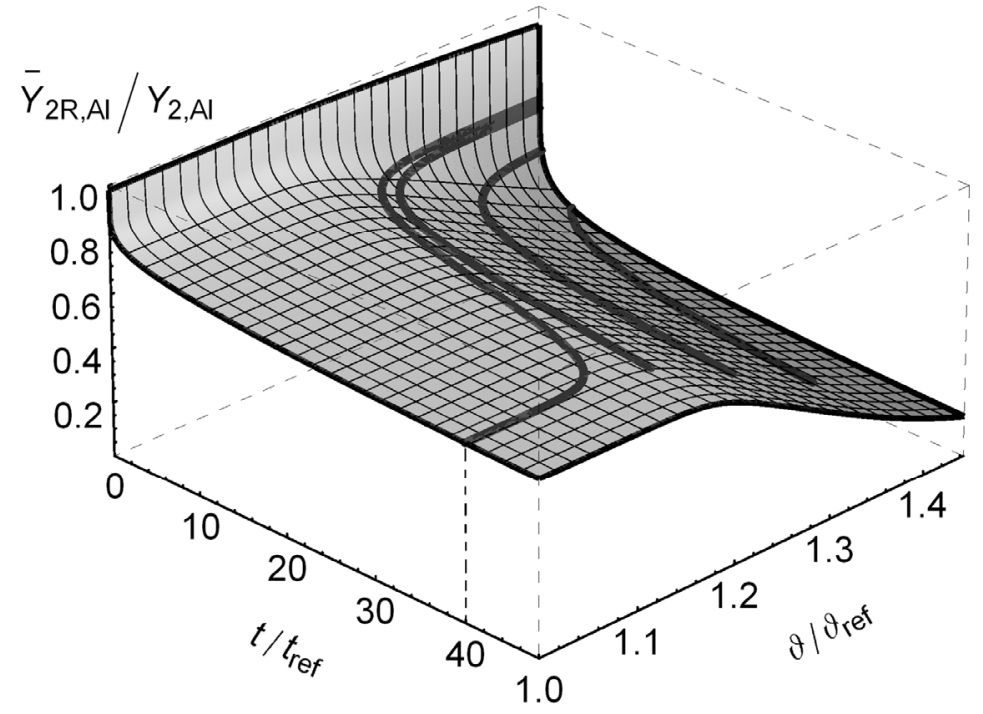

(a)

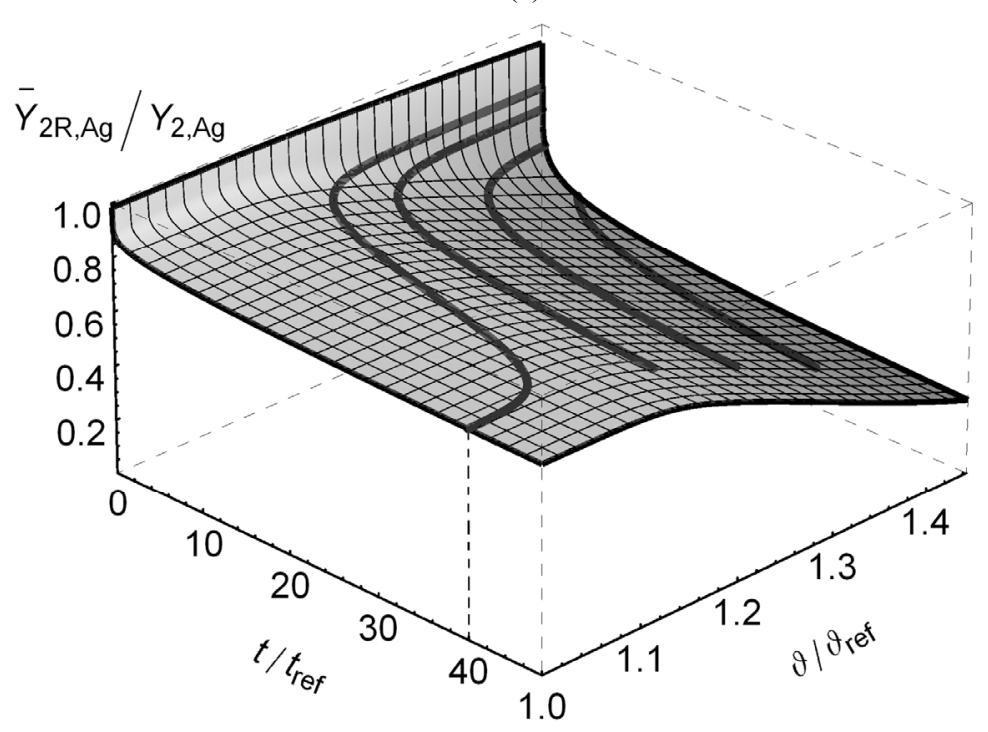

(b)

Fig. 5 Time-temperature equivalent surface for: (a) Al-seals; and (b) Ag-seals, where $Y_{2, A l}=386 \mathrm{~N} / \mathrm{mm}, Y_{2, A g}=494 \mathrm{~N} / \mathrm{mm}$, $t_{\text {ref }}=1$ year and $\vartheta_{\text {ref }}=293 \mathrm{~K}$. 
Substituting $\alpha_{E}\left(\theta_{i}\right)$ in Eq. (2) with $\alpha_{N}(\zeta)$ from Eq. (8), Eq. (2) can be rewritten as:

$$
\bar{Y}_{2 R}=Y_{2} \cdot \exp \left[\ln \left(\frac{\bar{Y}_{r e f, E}\left(\zeta_{r e f}\right)}{Y_{2}}\right)\left(\alpha_{N}(\zeta) \tau\right)^{1-\bar{m}}\right]
$$

with $\zeta_{\text {ref }}=1$. Comparing with Eq. (2), both time and temperature are continuous variables in Eq. (9).

The corresponding seal force values in time for arbitrary constant temperature values in the test range can be estimated from Eq. (9). A three-dimensional plot of the normalized restoring seal force function (time-temperature equivalent surface of restoring seal force of investigated metal seals) with time and temperature from Eq. (9) and Eq. (8) is depicted in Fig. 5 for Al- and Ag-seals. Therein, the thick lines at the surface represent restoring seal force values as isolines determined from forecasts at the point in time of 40 years at different constant temperatures of $293 \mathrm{~K}$, $348 \mathrm{~K}, 373 \mathrm{~K}$ and $398 \mathrm{~K}$. Furthermore, the same restoring seal force value can be derived for different temperatures based on the time-temperature equivalent principle. The presented application of Equations (8) and (9) is derived and valid for constant temperature conditions. Consequently, a change in temperature over time is not included in this approach.

\section{Conclusion}

In this work, a modified Arrhenius equation proposed by Nakamura et al. [6] was extended and applied for the calculation of time-temperature shift factors of arbitrary temperature values in a specific test range with suitable results considering the time-temperature superposition principle and based on an 'enhanced power-law model' developed as a master function for describing the relaxation behavior of metal seals. Thus, a non-linear interpolation of discrete points from experimental data was described by a continuous function in a temperature range which is representative for applied temperatures of metal seals in practice. Furthermore, a relaxation model was developed and applied with the time-temperature superposition principle for a specific metal seal type. Therewith, corresponding values of the restoring seal force under the influence of time and under arbitrary constant temperatures in the studied range were estimated with the developed analytical function. The introduced method is generally valid and widely applicable for issues which involve those time and temperature related effects.

\section{References}

[1] Qiao, L., Herbrich, U., Nagelschmidt, S., Zencker, U., and Völzke, H. 2016. "Modelling Long-term Relaxation of Metal Seals for Transport and Storage Casks." Presented at EMI 2016 International Conference, Metz, France, October 25-27, 2016.

[2] Zencker, U., Qiao, L., and Völzke, H. 2015. "Strategies for Numerical Modelling of Metal Gaskets in Transport and Storage Casks." Presented at Radioactive Materials Transport and Storage Conference and Exhibition, Oxford, United Kingdom, May 19-21, 2015.

[3] Völzke, H., Wolff, D., Probst, U., Nagelschmidt, S., and Schulz, S. 2013. "Long-term Performance of Metal Seals for Transport and Storage Casks." Presented at 17th Int. Symposium on the Packaging and Transportation of Radioactive Materials (PATRAM 2013), San Francisco, CA USA, August 18-23, 2013.

[4] Probst, U., Schulz, S., Jaunich, M., Wolff, D., and Völzke, H. 2014. "Langzeituntersuchungen an Metalldichtungen für Transport- und Lagerbehälter für Radioaktive Stoffe, Dichtungstechnik." Jahrbuch 2014, 162-73. ISGATEC GmbH, Mannheim. (in German)

[5] Nagelschmidt, S., Herbrich, U., Probst, U., and Wolff, D. 2014. "Evaluation of Test Results with Regard to Ageing Mechanisms of Metal Seals in Casks for Dry Storage of Spent Nuclear Fuel." Ageing of Materials \& Structures 2014 (AMS14), Paper No. 217757. Delft, The Netherlands.

[6] Nakamura, K., Takayanagi, T., and Sato, S. 1989. "A Modified Arrhenius Equation." Chemical Physics Letters 160 (3): 295-8.

[7] Kohlrausch, F. 1863. "Experimental-Untersuchungen über die Elastische Nachwirkung bei der Torsion, Ausdehnung und Biegung." Annalen der Physik und Chemie 7: 337-68. (in German)

[8] Williams, G., and Watts, D. C. 1970. "Non-symmetrical Dielectric Behavior Arising from a Simple Empirical Decay Function." Tran. Faraday Soc. 66: 80-5.

[9] Arrhenius, S. 1889. "On the Reaction Rate of the 

Equivalence in Relaxation Analysis of Metal Seals

Inversion of Non-refined Sugar upon Souring." Z. Phys. Chem. 4: 226-48.
[10] Laidler, K. J. 1984. "The Development of the Arrhenius Equation." Journal of Chemical Education 61 (6): 494-8. 